\title{
The Adaptive Frequency Band for Blood Pressure Variability Measurement during Nonstationary Conditions
}

\section{Chia-Chi Chang ${ }^{1,2,3}$, Tzu-Chien Hsiao ${ }^{1,3,4}$ and Hung-Yi Hsu ${ }^{5 *}$}

${ }^{1}$ Biomedical Electronics Translational Research Center, National Chiao Tung University, Taiwan, R.O.C

${ }^{2}$ Department of Electronics Engineering \& Institute of Electronics, National Chiao Tung University, Taiwan, R.O.C

${ }^{3}$ Institute of Biomedical Engineering, National Chiao Tung University, Taiwan, R.O.C

${ }^{4}$ Department of Computer Science, National Chiao Tung University, Taiwan, R.O.C

${ }^{5}$ Section of Neurology, Department of Internal Medicine, Tungs' Taichung Metro Harbor Hospital, Taichung, Taiwan, R.O.C

\begin{abstract}
Background: The variation of blood pressure indicates the status of cardiovascular circulation. The spectral analysis of blood pressure variability (BPV) provides a way to quantitatively assess the variations by specific fixed frequency band. Blood pressure contains various non-stationary fluctuations and varies individually. It is hard to assess the non-stationary characteristics based on stationary method.
\end{abstract}

Method: Recently, a novel adaptive extraction method, called empirical mode decomposition (EMD), was proposed and is capable to extract the non-stationary intrinsic trends from blood pressure waveform. Results: The results showed that the non-stationary intrinsic trends extracted by EMD are high correlated to the power in conventional fixed frequency band $(r>0.7)$.

Conclusions: This study examined the potential usage of EMD on BPV measurement and provided the reliable estimation of BPV based on adaptive frequency band. Moreover, the main frequency of the non-stationary trend can be evaluated by this method. It helps for cardiovascular studies and the optimal frequency band exploration.

Keywords: Blood pressure variability (BPV); Arterial blood pressure (ABP); Empirical mode decomposition (EMD); Intrinsic mode function (IMF)

\section{Introduction}

The blood pressure is an important indicator of cardiovascular circulation. Its trend, including short-term (10 minutes) and longterm (24 hours), indicates several reflex of auto-regulation [1]. The blood pressure variability (BPV) is a reliable technique for quantitative assessment of the status of auto-regulation in cardiovascular circulation $[2,3]$. BPV is considered as a novel risk factor for cardiovascular disease and its assessment and quantification is of physiopathological and prognostic importance [4,5]. Conventional BPV adopted spectral analysis, based on fast Fourier transform (FFT) or discrete wavelet transform (DWT), for the measurement of the magnitude at each frequency band $[6,7]$. Lots of clinical BPV studies investigated that each frequency band indicated different neurohumoral systems mechanisms involved in non-stationary dynamic auto-regulation. Very low frequency fluctuations (VLF, $0.01-0.04 \mathrm{~Hz}$ ) indicate myogenic vascular function $[8,9]$. Low frequency fluctuations $(\mathrm{LF}, 0.04-0.15 \mathrm{~Hz}$ ) indicate the sympathetic nervous activities, and high frequency fluctuations $(\mathrm{HF}, 0.15-0.4 \mathrm{~Hz})$ indicate parasympathetic nervous activities and the release of endothelial-derived nitric oxide [3].

In order to get much reliable and precise assessment, the division of the frequency band becomes an important issue of BPV research. Since the optimal frequency band for the assessment of auto-regulation is still unknown, conventional BPV studies adopted different definitions of the frequency band division. It limits the reliability and utility of BPV in clinic. Recently, a novel adaptive method, called empirical mode decomposition (EMD), was proposed [10]. EMD is a specific filter bank that can extract the non-stationary intrinsic fluctuations adaptively without information loss. EMD has potential to extract the non-stationary trend, called intrinsic mode function (IMF), at each time scale, including short-term and long-term $[11,12]$. Though there were several studies examined that the potential usage of EMD in BPV analysis, the evaluation was still limited by the time resolution of beat-tobeat blood pressure [13]. It had been examined that the non-stationary trend can be extracted by EMD and be quantitatively assessed by the spectral integration method, including the calculation of averaged period and energy density [14]. By using this method, the assessment of BPV becomes adaptive, called adaptive BPV, which is based on adaptive trend extraction rather than the specific fixed frequency band. But its reliability needs to be examined by the comparison with conventional BPV.

The aim of the study is to 1) extract the non-stationary intrinsic short-term fluctuations at each time scale; 2) examine the reliability of the extracted fluctuations by different physiological challenges; and 3) examine the reliability compared to the conventional BPV method and to examine the changes of main frequency during different physiological challenges.

\section{Experiment and Method}

\section{Subjects and data collection}

All measurements were performed in a temperature-controlled room with the subjects lying supine on a tilting table which was tilted up automatically. All measurements were conducted in the morning, at least $2 \mathrm{~h}$ subsequent to the patient having eaten a light breakfast. The beat-to-beat arterial blood pressure (ABP) were recorded by Task Force $^{\oplus}$ Monitor equipped with a servo-controlled plethysmography (CNSystems, Medizintechnik AG, Graz, Austria) at a sampling rate of $200 \mathrm{~Hz}$, and saved on the hard disc for later analysis.

*Corresponding author: Hung-Yi Hsu, Section of Neurology, Department of Internal Medicine, Tungs' Taichung Metro Harbor Hospital, No. 699, Sec. 8, Taiwan Blvd, Wuci District, Taichung 435, Taiwan R.O.C, Tel: +886-9-10597960; E-mail: hungyihsu@gmail.com

Received November 11, 2014; Accepted December 15, 2014; Published December 18, 2014

Citation: Chang CC, Hsiao TC, Hsu HY (2014) The Adaptive Frequency Band for Blood Pressure Variability Measurement during Nonstationary Conditions. J Pulm Respir Med 5: 230. doi:10.4172/2161-105X.1000230

Copyright: (c) 2014 Chang CC, et al. This is an open-access article distributed under the terms of the Creative Commons Attribution License, which permits unrestricted use, distribution, and reproduction in any medium, provided the original author and source are credited. 
Fifteen healthy subjects (6 males, aged $43 \pm 17$ years old) participated in this study without any history of cardiovascular diseases. All recruited subjects were asked to rest quietly in supine position for 10 minutes. While all recruited signals were stabilized, a 10-min baseline recording was performed under spontaneous breathing. Then, all recruited subjects were asked to breathe following the examiner's verbal instruction at a rate of 6 cycles per minute and at 30 cycles per minute. Each test was performed for 2 minutes. There was a 5-min rest between each test. At last, participants were tilting up passively on the automatic tilting table and kept in the tilt-up position for 10 minutes. This study was approved by institutional review board of the hospital. Informed consent was obtained from all subjects before the experiment.

\section{Empirical mode decomposition}

The IMFs are extracted by iterative detrending process, called sifting process. The estimated trend at each time scale was determined by the mean of upper envelope and lower envelope at each iteration. The sifting process of EMD contains several steps. The upper envelope $U(t)$ and lower envelope $L(t)$ were generated by cubic spline interpolation with all local maxima and all local minima. Both envelopes covered $x(t)$ as the specific time scale. The trend was calculated by the average of $U(t)$ and $L(t)$.

$$
m(t)=(U(t)+L(t) / 2
$$

The first intermediate component $h_{1}(t)$ was calculated by subtracting the trend from original time series $x(t)$, as the detrending process.

$$
h_{1}(t)=x(t)-m_{1}(t)
$$

And the subtraction was iteratively performed

$$
\left\{\begin{array}{c}
h_{1}(t)=x(t) \\
h_{1 k}(t)=h_{1(k-1)}(t)-m_{1 k}(t) k>1
\end{array}\right.
$$

$h_{1 k}(t)$ was considered as an IMF only if the kth trend $m_{1 k}(t)$ satisfied the criterion as the steady constant trend.

$$
h_{1 k}(t)=I M F_{1}(t)
$$

The first residue was also calculated.

$$
r_{1}(t)=x(t)-I M F_{1}(t)
$$

The residue $r_{i}(t)$ was the target signal of the ith iteration of EMD, as $x(t)$ performed at the first iteration. After $\mathrm{n}$ iteration, $x(t)$ was decomposed into $\mathrm{nIMFs}, I M F_{1}(t) \sim I M F_{n}(t)$, and one residue, $r_{n}(t)$, which was either the steady trend or a constant.

$$
x(t)=\sum_{i=1}^{n} I M F_{i}(t)+r_{n}(t)
$$

EMD decomposes the non-stationary data into a finite set of IMFs without information loss or distortion. The analysis system used in this study was developed by using a commercial programming platform (LabVIEW, version 2011, National Instruments Corp., Austin, TX, USA).

\section{Averaged frequency and energy density}

It had been examined that the non-stationary characteristics of IMFs can be assessed by the spectral integration method with averaged period and corresponding energy density [14]. This method was originally used to study the characterization of intrinsic white noise by evaluating the distribution of IMFs' logarithmic averaged period and corresponding logarithmic energy density [15].

$$
E_{n}=\frac{1}{N} \sum_{t=1}^{N}\left[I M F_{n}(t]^{2}\right.
$$

$$
\bar{T}_{n}=\int S_{\mathrm{l} T, n} d \mathrm{~h} T\left(\int S_{\mathrm{h} T, n} \frac{d \mathrm{~h} T}{T}\right)^{-1}
$$

$E_{n}$ is the energy density of the nth IMF and $S_{\mathrm{h} T, n}$ is the Fourier spectrum of the nth IMF as the function of $h T$. $T$ is the period along the x-axis of the spectrum after changing variable, and $\bar{T}_{n}$ is the estimator of the averaged period of the nth IMF. In this study, the averaged frequency, as main frequency, was estimated by the inversion of averaged period.

\section{Spectral analysis of blood pressure variability}

The steps of BPV were shown in Figures 1 and 2. In conventional BPV, the peak detection algorithm was performed for systolic blood pressure (sBP) detection. After the beat-to-beat sBP series, as systogram, was got, the sBP time series was calculated from systogram by interpolation method [3]. The spectral analysis, such as FFT or DWT, was performed for quantitative assessment according to the specific fixed frequency band. In adaptive BPV based on EMD, the non-stationary intrinsic trends (IMF5-7 in this study) were extracted from ABP signal by EMD [14]. Each non-stationary intrinsic trend was assessed by the spectral analysis with averaged frequency and corresponding energy density for the comparison and the examination.

\section{Statistic analysis}

Paired-sample $t$ test was used to test the significance of the logarithmic averaged period and logarithmic energy density during 6-cycle breathing, 30-cycle breathing, and head-up tilting test (HUT) compared to baseline. $\mathrm{P}$ value of $<0.05$ was considered significant. Pearson's correlation coefficient ( $r$ ) was applied to evaluate the consistency of the results between convensional spectral analysis and adaptive spectral analysis. All statistical analysis was performed by using commercial statistics software (Statistical Package for Social Science, version 15.0, SPSS Inc., Chicago, Illinois).

\section{Results}

\section{Conventional blood pressure variability}

The spectral analysis of conventional BPV was summarized in Table 1. LF and HF increased during HUT. During paced respiration, VLF decreased significantly, while LF increased significantly. HF decreased during 6-cycle respiration and increased during 30-cycle respiration.

\section{Adaptive frequency band in blood pressure variability}

The spectral analysis of adaptive BPV, including its averaged frequency and corresponding energy density, was summarized in Table 2. During HUT, the energy density of IMF6 increased. During paced respiration with 6-cycle per minute, the energy density of IMF6 increased significantly while the energy density of IMF7 decreased significantly. During paced respiration with 30 -cycle per minute, the energy density of IMF5 increased significantly while the energy density of IMF6 and IMF7 decreased significantly. Moreover, the averaged frequency of IMF6 and IMF7 increased significantly during 30-cycle breathing.

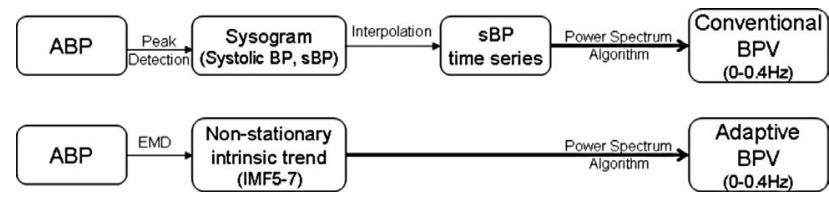

Figure 1: The flow chart of spectral analysis of BPV. The top graph presents the algorithm of the spectral analysis of conventional BPV and the bottom graph presents the algorithm of the spectral analysis of adaptive BPV. 


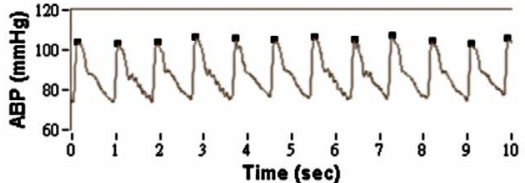

(a)
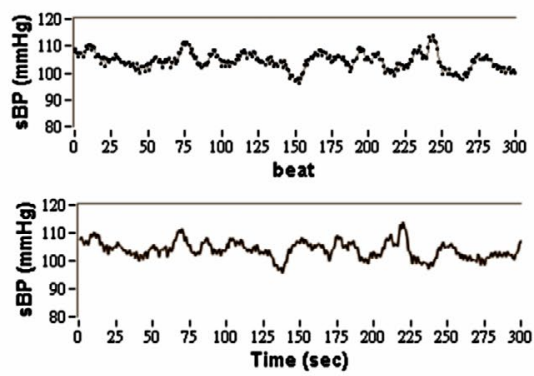

(c)

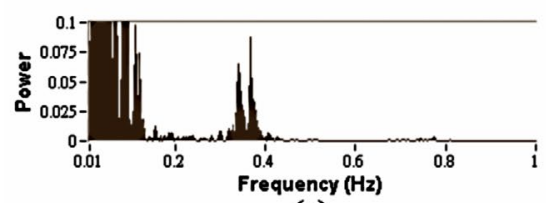

(e)

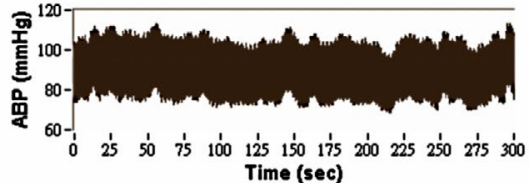

(b)

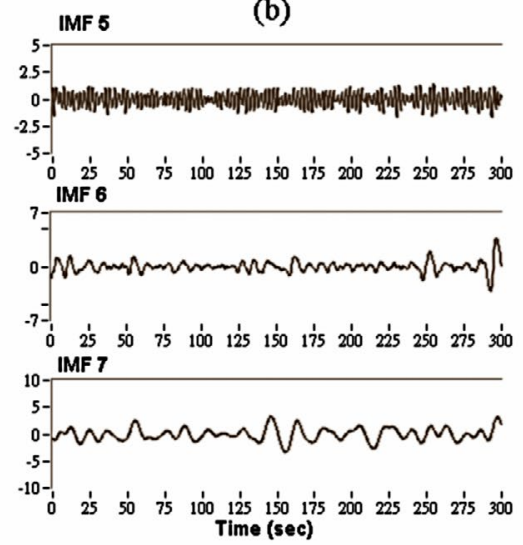

(d)

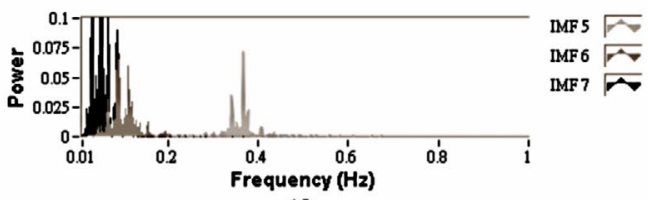

(f)

Figure 2: The illustration of spectral analysis of BPV, including (a) The ABP signal with systolic blood pressure (sBP) peak detection (solid dot) within 10 seconds (b) The ABP signal within 300 seconds (c) top graph showed the systogram and bottom graph showed sBP time series after interpolation (d) IMF5-7 of ABP signal (e) FFT Spectrum of sBP time series (f) FFT Spectrums of IMF5-7.

\begin{tabular}{|c|c|c|c|}
\hline & VLF & LF & HF \\
\hline Baseline & $2.75 \pm 2.11$ & $2.58 \pm 2.23$ & $0.56 \pm 0.53$ \\
\hline HUT & $2.50 \pm 2.91$ & $3.65 \pm 3.08$ & $0.83 \pm 1.04$ \\
\hline 6-cycle & $1.13 \pm 1.72 \S$ & $6.81 \pm 7.33^{*}$ & $0.30 \pm 0.22^{*}$ \\
\hline 30-cycle & $0.92 \pm 0.82 \S$ & $1.42 \pm 2.09^{*}$ & $1.25 \pm 1.93$ \\
\hline
\end{tabular}

${ }^{*} p<0.05$ compared with baseline; $\S$ means $p<0.01$ compared with baseline. The present form is (mean \pm standard deviation). VLF denotes very low frequency band $(0.01-0.04 \mathrm{~Hz})$; LF denotes low frequency band $(0.04-0.15 \mathrm{~Hz})$; HF denotes high frequency band $(0.15-0.4 \mathrm{~Hz})$; HUT denotes head-up tilt; 6 -cycle denotes paced respiration with 6 cycles per minute; 30-cycle denotes paced respiration with 30 cycles per minute.

Table 1: Spectral analysis of conventional short-term blood pressure variability (BPV).

\section{Discussion}

The spontaneous fluctuation of ABP indicates the auto-regulation in cardiovascular circulation. The frequency of these regulated fluctuations indicates the response of auto-regulation. The oscillation within specific frequency range presents specific physiological mechanisms with feedback control loop in specific physiological regulation scales. In order to assess the related regulation quantitatively, conventional BPV measurement adopted the spectral power of ABP time series within specific fixed frequency band. But the spectral analysis of conventional BPV might overestimate the power in fixed frequency band, such as during 6-cycle breathing for example. Besides, the conventional BPV adopted the interpolated $\mathrm{ABP}$ time series as the target signal, and the time resolution of the interpolated $\mathrm{ABP}$ time series is limited by the heart rate. The adaptive BPV provided a novel reliable indicator for main frequency peak exploration and quantitative assessment with accurate spectral power with uniform time scale. The results showed the consistent investigation between conventional BPV and adaptive BPV.

It had been demonstrated that EMD could act as a filter bank adaptively [16] without composited phase distortion, which existed in conventional band-pass filter. The results in this study extracted IMF5-7 as the non-stationary intrinsic trend of ABP signal. The energy density of IMF6 increased during HUT. The passive tilting table causes temporary orthostatic hypotension and induces the sympathetic nervous activities for the compensation mechanism [17]. The vascular system would involve in the compensation, which is caused by the feedback of the ABP at a number of baro-receptors through autonomic nervous system. The averaged frequency of IMF6 is around $0.11 \mathrm{~Hz}$, which is in the frequency range of LF and presents the sympathetic nervous activities. This result of energy density of IMF6 is consistent to LF in conventional BPV $(r=0.889)$.

The energy density of IMF6 increases during 6-cycle breathing. The paced respiration with 6 cycles per minute would cause the major intrinsic fluctuation of ABP around $0.1 \mathrm{~Hz}$, so-called Traube-Hering waves (Mayer waves) [18], which is a major ABP fluctuation caused by respiration in the thorax $(>0.2 \mathrm{~Hz})$ and originated from the change in venous return owing to the change in intra-thoracic pressure. The main frequency can also be evaluated by the averaged frequency, which is around $0.11 \mathrm{~Hz}$ in IMF6. This result of energy density of IMF6 is consistent to LF in conventional BPV $(r=0.951)$. The energy density of IMF5 increases during 30-cycle breathing. The paced respiration with 30 cycles per minute is hyperventilation and would cause the major 
Citation: Chang CC, Hsiao TC, Hsu HY (2014) The Adaptive Frequency Band for Blood Pressure Variability Measurement during Nonstationary Conditions. J Pulm Respir Med 5: 230. doi:10.4172/2161-105X.1000230

Page 4 of 5

\begin{tabular}{|c|c|c|c|c|c|c|}
\hline & \multicolumn{3}{|c|}{ Averaged frequency } & \multicolumn{2}{c|}{ Energy density } \\
\hline & IMF5 & IMF6 & IMF7 & IMF5 & IMF6 \\
\hline Baseline & $0.40 \pm 0.10$ & $0.11 \pm 0.02$ & $0.05 \pm 0.01$ & $33.00 \pm 34.13$ & $48.14 \pm 64.6$ & $124.84 \pm 103.97$ \\
\hline HUT & $0.37 \pm 0.07$ & $0.11 \pm 0.03$ & $0.05 \pm 0.01$ & $17.04 \pm 11.20$ & $76.51 \pm 100.41$ & $116.04 \pm 94.23$ \\
\hline 6-cycle & $0.49 \pm 0.19$ & $0.11 \pm 0.01$ & $0.08 \pm 0.02$ & $32.10 \pm 51.48$ & $278.50 \pm 292.66 \S$ & $53.51 \pm 34.08^{*}$ \\
\hline 30-cycle & $0.41 \pm 0.06$ & $0.14 \pm 0.04 \S$ & $0.06 \pm 0.01 \S$ & $96.63 \pm 94.59^{*}$ & $12.22 \pm 10.01^{*}$ & $35.04 \pm 43.24 \S$ \\
\hline
\end{tabular}

${ }^{*} p<0.05$ compared with baseline; $\S$ means $p<0.01$ compared with baseline.

The present form is (mean \pm standard deviation). VLF denotes very low frequency band $(0.01-0.04 \mathrm{~Hz}$ ); LF denotes low frequency band ( $0.04-0.15 \mathrm{~Hz})$; $\mathrm{HF}$ denotes high frequency band $(0.15-0.4 \mathrm{~Hz})$; IMF denotes intrinsic mode function; HUT denotes head-up tilt; 6 -cycle denotes paced respiration with 6 cycles per minute; 30 -cycle denotes paced respiration with 30 cycles per minute.

Table 2: Spectral analysis of adaptive short-term blood pressure variability (BPV) based on EMD.

Conventional BPV

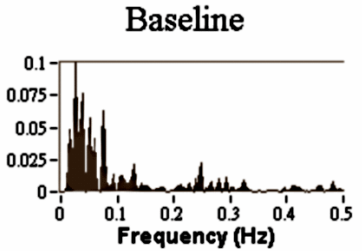

(a)

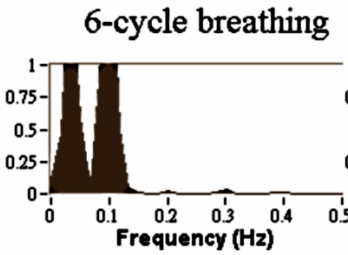

(b)

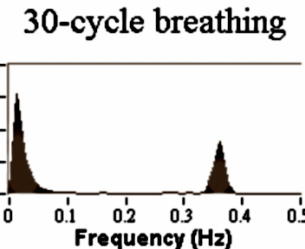

(c)

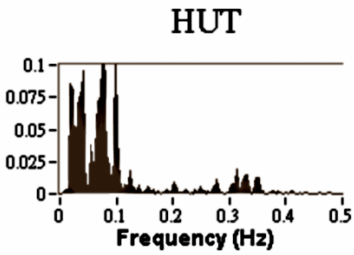

(d)

Adaptive BPV

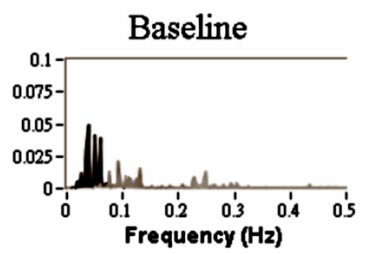

(e)

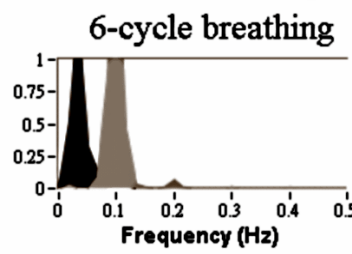

(f)

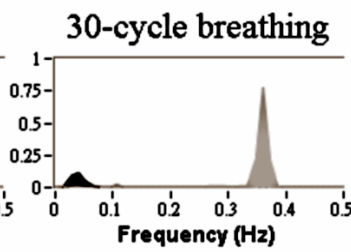

(g)

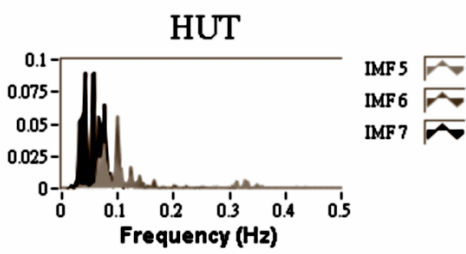

(h)

Figure 3: The illustration of BPV spectrum during different physiological challenges, including (a) baseline, (b) 6 cycles breathing per minute, (c) 30 cycles breathing, (d) head-up tilt (HUT) by using conventional BPV, and (e) baseline, (f) 6 cycles breathing per minute, (g) 30 cycles breathing per minute, (h) HUT by using adaptive BPV.

intrinsic fluctuation of $\mathrm{ABP}$ around $0.5 \mathrm{~Hz}$ as Mayer wave. This result of energy density of IMF5 is consistent to HF in conventional BPV $(\mathrm{r}=0.733)$. On the other hand, the energy density of IMF6 and IMF7 decreases owing to the domination of Mayer wave, which is consistent to the change in LF and VLF in conventional BPV. The other intrinsic fluctuations of ABP are regulated by sympathetic nervous system, such as the changes in the peripheral resistance $(0.05-0.2 \mathrm{~Hz})$, or regulated by the temperature regulation $(0.01-0.05 \mathrm{~Hz})[7-9]$.

The spectrum of BPV in each physiological challenge is summarized in Figure 3. Most of the optimal frequency bands of different physiological mechanisms are still unclear and the main frequency of specific regulation might be different individually. Moreover, during physiological challenge, the non-stationary intrinsic oscillation of ABP does not have a fixed period and each center frequency can vary considerably. Since the spectral components are usually cross band in frequency domain and the main frequency of the non-rhythmic eventdriven regulation would shift in non-stationary condition, it is much difficult to explore the regulation phenomenon with specific fixed frequency band.

The method in this study demonstrated the potential usage of EMD for BPV measurement, and provided the adaptive estimation with main frequency evaluation. This study has some limitations. First, the resolution of the averaged frequency is limited by the sampling rate of $A B P$ signal acquisition, and the bound of averaged frequency is restricted by the length of total recording period. The lower frequency band, such as VLF $(0.01-0.04 \mathrm{~Hz})$, the study explored, the longer length of the recording period required. Second, the non-stationary intrinsic trend extraction based on EMD mainly depends on the selective parameters. Only few researchers studied the IMF with the same definition. Although this study examined the potential BPV assessment with adaptive frequency band and main frequency estimation, the ideal non-stationary indicators of cardiovascular auto-regulation need further exploration.

\section{Conclusions}

This study showed that the BPV measurement with adaptive frequency band provided consistent results with conventional BPV. The adaptive frequency band provides another option for quantitative assessment of BPV without any fundamental assumption, such as the definition of specific fixed frequency band. Moreover, the adaptive $\mathrm{BPV}$ in this study provided much more information, such as the main frequency estimation. The adaptive BPV helps for the optimal frequency exploration and cardiovascular auto-regulation study.

\section{Acknowledgment}

This work was fully supported by the Taiwan Ministry of Science and Technology under grant numbers MOST-103-2218-E-009-016, MOST-103-2221-E-009-139 and MOST-103-2220-E-009-006 and in part by "Aim for the Top University Plan" 
Citation: Chang CC, Hsiao TC, Hsu HY (2014) The Adaptive Frequency Band for Blood Pressure Variability Measurement during Nonstationary Conditions. J Pulm Respir Med 5: 230. doi:10.4172/2161-105X.1000230

of the National Chiao Tung University and Ministry of Education, Taiwan, R.O.C. This work was also supported in part by the UST-UCSD International Center of Excellence in Advanced Bioengineering sponsored by the Taiwan National Science Council I-RiCE Program under grant number NSC-101-2911-I-009-101.

\section{References}

1. Grassi G, Bombelli M, Brambilla G, Trevano FQ, Dell'oro R, et al. (2012) Tota cardiovascular risk, blood pressure variability and adrenergic overdrive in hypertension: evidence, mechanisms and clinical implications. Curr Hypertens Rep 14: 333-338.

2. Andriessen P, Schoffelen RL, Berendsen RC, de Beer NA, Oei SG, et al. (2004) Noninvasive assessment of blood pressure variability in preterm infants. Pediatr Res 55: 220-223.

3. Parati G, Bilo G, Valentini M (2008) Blood pressure variability: methodological aspects, pathophysiological and clinical implications. Manual of Hypertension of the European Society of Hypertension, Mancia G, Grassi G and Kjeldsen SE, Eds. 61-71.

4. Stauss HM (2007) Identification of blood pressure control mechanisms by power spectral analysis. Clin Exp Pharmacol Physiol 34: 362-368.

5. Parati G (2005) Blood pressure variability: its measurement and significance in hypertension. J Hypertens Suppl 23: S19-25.

6. Parati G, Ochoa JE, Lombardi C, Bilo G (2013) Assessment and management of blood-pressure variability. Nat Rev Cardiol 10: 143-155.

7. Stauss HM (2007) Identification of blood pressure control mechanisms by power spectral analysis. Clin Exp Pharmacol Physiol 34: 362-368.

8. Tzeng YC, Chan GS, Willie CK, Ainslie PN (2011) Determinants of human cerebral pressure-flow velocity relationships: new insights from vascular modelling and $\mathrm{CaA} \hat{A}^{0}{ }^{\circ}$ channel blockade. J Physiol 589: 3263-3274.
9. Tan CO, Hamner JW, Taylor JA (2013) The role of myogenic mechanisms in human cerebrovascular regulation. J Physiol 591: 5095-5105.

10. Huang NE, Shen Z, Long SR, Wu MC, Shih SS, et al. (1998) The empirical mode decomposition and the Hilbert spectrum for nonlinear and non-stationary time Series analysis. Proc R Soc Lond A 454: 903-995.

11. Chang CC, Hsu HY, Hsiao TC (2013) Quantitative non-stationary assessment of cerebral hemodynamics by empirical mode decomposition of cerebral Doppler flow velocity. Adv Adapt Data Anal 5: 1350002.

12. Yeh JR, Lin TY, Shieh JS, Chen Y, Huang NE, et al. (2008) Investigating complex patterns of blocked intestinal artery blood pressure signals by empirical mode decomposition and linguistic analysis. J Phys Conf Ser 96: 012153.

13. de Souza Neto EP1, Abry P, Loiseau P, Cejka JC, Custaud MA, et al. (2007) Empirical mode decomposition to assess cardiovascular autonomic control in rats. Fundam Clin Pharmacol 21: 481-496.

14. Chang CC, Hsiao TC, Hsu HY (2014) Depicting respiratory characteristics of blood pressure signal by using empirical mode decomposition. J Pulm Respi Med 4: 209.

15. Wu Z, Huang NE (2004) A study of the characteristics of white noise using the empirical mode decomposition method. Proc R Soc Lond A 460: 1597-1611.

16. Flandrin P, Rilling G, Goncalves $P$ (2004) Empirical mode decomposition as a filter bank. IEEE Signal Process Lett 11: 112-114.

17. Malliani A, Pagani M, Lombardi F, Cerutti S (1991) Cardiovascular neura regulation explored in the frequency domain. Circulation 84: 482-492.

18. de Jong W (2000) Blood pressure variability in neonates. With a special focus on signal acquisition and signal processing. PhD Thesis, Eindhoven University of Technology Eindhoven 3-6. 